\title{
Amyloid-beta and phosphorylated tau in post-mortem Alzheimer's disease retinas
}

\author{
Jurre den Haan ${ }^{1 *}\left(\mathbb{D}\right.$, Tjado H. J. Morrema ${ }^{2 \dagger}$, Frank D. Verbraak ${ }^{3}$, Johannes F. de Boer ${ }^{4}$, Philip Scheltens ${ }^{1}$, \\ Annemieke J. Rozemuller ${ }^{2}$, Arthur A. B. Bergen ${ }^{5,6}$, Femke H. Bouwman ${ }^{1}$ and Jeroen J. Hoozemans ${ }^{2}$
}

\begin{abstract}
In-vivo labeling of retinal amyloid-beta(A $\beta$ ) and tau has potential as non-invasive biomarker for Alzheimer's disease (AD). However, literature on the presence of $A \beta$ and phosphorylated tau (pTau) in AD retinas is inconclusive. We therefore assessed the presence of $A \beta$ and pTau in post-mortem retinas in $6 A D$ and 6 control cases who donated brains and eyes to the Netherlands Brain Bank. Neuropathological diagnosis of AD was made according to NIA-AA criteria. Formalin fixed retinas were dissected in quadrants and cross-sections of medial and superior retinas were made. Immuno-histochemical stainings were performed for AB, amyloid precursor protein (APP) and pTau. To assess translation to an in-vivo set up using curcumin as labelling fluorophore, co-stainings with curcumin were performed. No typical Aß-plaques and neurofibrillary tangles, like in the cerebral cortex, were observed in $A D$ retinas. A diffuse immunoreactive signal for pTau was increased in the inner and outer plexiform layers of the retina in $\mathrm{AD}$ cases compared to control cases with absence of cerebral amyloid pathology. Immunostaining with anti-A $\beta$ and anti-APP antibodies yielded signal in ganglion cells, amacrine cells, horizontal cells and Müller cells in both control and AD cases. We observed small extracellular deposits positive for anti-A 3 antibodies 12F4 and 6E10 and negative for 4G8 and curcumin. A subset of these deposits could be characterized as corpora amylacea. In conclusion we found that retinal manifestations of AD pathology appear to be different compared to cerebral AD pathology. Using a qualitative cross-sectional approach, we did not find AB/APP related differences in the retina between AD and control subjects. In contrast, tau related changes were found to be present in cases with cerebral AD pathology, suggesting retinal tau as a potential biomarker for AD.
\end{abstract}

Keywords: Retina, Amyloid, Tau, Post-mortem, Alzheimer's disease, Neurodegeneration

\section{Introduction}

Alzheimer's disease (AD) is the leading cause of dementia [1] and its pathology develops 15-20 years before symptom onset [19]. Key pathological hallmarks of AD are neuronal loss, extra-cellular accumulation of amyloid-plaques containing amyloid-beta $(A \beta)$ and neurofibrillary tangles containing tau [6]. In addition, cerebral amyloid angiopathy (CAA) [33] and neuro-inflammation [13] are recognized as important events involved in AD pathology. The retina, embryologically derived from the diencephalon

\footnotetext{
* Correspondence: j.denhaan1@vumc.nl

† Jurre den Haan and Tjado H. J. Morrema contributed equally to this work. ${ }^{1}$ Department of Neurology, Alzheimer Center Amsterdam, Amsterdam Neuroscience, Vrije Universiteit Amsterdam, Amsterdam UMC, Mailbox 7057, Amsterdam 1007 MB, The Netherlands

Full list of author information is available at the end of the article
}

and sharing many features with brain tissue, is hypothesized to serve as a 'window to the brain', as neuropathological changes in retinal neurons might mirror brain pathology [26]. Non-invasive retinal imaging is routinely performed in ophthalmology clinics with high resolution imaging $( \pm 5 \mu \mathrm{m})$. Fluorescent imaging of specific proteins is on the horizon and might serve as a non-invasive diagnostic tool to visualize molecular processes involved in ophthalmological and neurological disease $[5,7,22]$.

Previous post-mortem studies have investigated the presence of pathological changes in human retinas in $\mathrm{AD}$, however the detection of these changes was not consistent across these studies [20]. On the one hand, neuronal thinning, ganglion cell loss and tau deposition are reported $[3,30]$. On the other hand, $A \beta$ deposition was found in whole mounted retinas and cross-sections by three studies from one lab, preferentially affecting the

(c) The Author(s). 2018 Open Access This article is distributed under the terms of the Creative Commons Attribution 4.0 International License (http://creativecommons.org/licenses/by/4.0/), which permits unrestricted use, distribution, and 
superior retina [22-24]. Recently, results from an in-vivo study showed an increase in retinal fluorescence after curcumin supplementation in $\mathrm{AD}$ patients, hypothesized to reflect retinal amyloid [22]. However, $A \beta$ presence in the retina still remains controversial as three other groups were unable to replicate this finding in $\mathrm{AD}$ retinas $[14,30,34]$.

Possible explanations for these discrepancies are differences in methodology, as well as interpretation of findings. First, different anti-A $\beta$ antibodies were used (including 6E10, 12F4, 6F3D and 4G8). Second, different methods of processing and dissection of retinas were applied, as some groups prepared retinal crosssections while others generated flat mounts. A crosssectional approach yields morphological information on layer distribution of the pathology, at the cost of sampling bias, due to the limited surface covered. In contrast, using flat mounts, a large surface of the retina is studied, yet lacking layer information. Lastly, with the predilection of pathology in the superior retina reported [22], it might be that other groups assessed parts of the retina with less pathological changes.

To better understand retinal involvement in AD pathology, we explored the presence of AD pathology with different antibodies in post-mortem retinal and brain tissue of patients with a definite post-mortem diagnosis of $\mathrm{AD}$, in order to assess molecular targets for in vivo (fluorescent) retinal imaging in AD.

\section{Materials and methods}

\section{Subjects}

We assessed retinal tissue of $6 \mathrm{AD}$ patients and 6 controls who donated their brain and eyes to the Dutch Brain Bank between 2010 and 2015. Neuropathological diagnosis of $\mathrm{AD}$ was made following NIA-AA criteria including Braak and Braak-staging for neurofibrillary tangles and amyloid, and CERADstaging for neuritic plaques [4, 17]. We selected cases with a clinical diagnosis of $\mathrm{AD}$ and neuropathological staging of Braak $\geq 4$. Controls were adults without cognitive decline and Braak $\leq 2$. We excluded cases with other neurodegenerative diseases based on history or post-mortem diagnosis. Cohort characteristics are shown in Table 1.

\section{Tissue processing}

Within $12 \mathrm{~h}$ post-mortem, eyes were removed. The anterior parts of the eye, including the cornea and lens, were dissected and the eyecup was filled with tissue-tek (cat\# 4583, Sakura). Eyes were snap frozen using iso-pentane at $-100^{\circ} \mathrm{C}$ and stored at $-80^{\circ} \mathrm{C}$. Eyes were defrosted in 4\% PFA at room temperature for $48 \mathrm{~h}$ prior to dissection. The eye was dissected in four quadrants through the vertical and horizontal meridian resulting in
Table 1 Cohort characteristics

\begin{tabular}{llllll}
\hline$\#$ & Pathological diagnosis & Sex & Age & Braak & Amyloid \\
\hline 1 & AD & F & 73 & VI & C \\
2 & AD & M & 70 & VI & C \\
3 & AD & M & 56 & V & C \\
4 & AD & F & 91 & V & C \\
5 & AD & F & 95 & IV & C \\
6 & AD & F & 89 & IV & C \\
7 & HC & F & 89 & $\|$ & B \\
8 & HC & M & 80 & $\|$ & I \\
9 & HC & F & 76 & $\|$ & O \\
10 & HC & F & 79 & $\|$ & O \\
11 & HC & F & 57 & 0 & A \\
12 & HC & M & 49 & 0 & O \\
\hline AD Azher's & & & &
\end{tabular}

$A D$ Alzheimer's disease, $H C$ Healthy control

naso-superior, naso-inferior, temporal-superior and temporal-inferior quadrants containing retinal tissue from macula to ora serrata (Fig. 1). Quadrants were dehydrated prior to embedding in paraffin according to the following protocol: $3 \mathrm{~h}$ formalin $4 \%$ at $35^{\circ} \mathrm{C}, 1 \mathrm{~h}$ ethanol $70 \% 35^{\circ} \mathrm{C}, 1 \mathrm{~h}$ ethanol $80 \% 35^{\circ} \mathrm{C}, 1 \mathrm{~h}$ ethanol $96 \% 35^{\circ} \mathrm{C}$, 3 times $1 \mathrm{~h}$ alcohol $100 \% 35^{\circ} \mathrm{C}$, 3 times $1 \mathrm{~h}$ xylene $35^{\circ} \mathrm{C}$, 4 times $1 \mathrm{~h}$ paraffin $62^{\circ} \mathrm{C}$. Paraffin embedded tissue was sectioned using a microtome at $5-\mu \mathrm{m}$ and $10-\mu \mathrm{m}$ thickness and mounted on TOMO slides (cat\# TOM-1190, Matsunami). Mounted slides were dried overnight at $37^{\circ} \mathrm{C}$ prior to staining. Per patient, at least 25 sections per region were stained with $\mathrm{APP} / \mathrm{A} \beta$ antibodies to overcome sampling bias.

\section{Immunohistochemistry (IHC)}

Immunohistochemistry was initially performed on 5 and $10 \mu \mathrm{m}$ thick eye sections. As both thicknesses yielded comparable results, $10 \mu \mathrm{m}$ sections were therefore used for the full cohort. Sections were deparaffinized and endogenous peroxidase activity was suppressed with $0.3 \%$ $\mathrm{H}_{2} \mathrm{O}_{2}$ in phosphate buffered saline (PBS) for $30 \mathrm{~min}$. Antigen retrieval was performed with $10 \mathrm{mM} / \mathrm{L}$ pH 6.0 sodium citrate buffer heated by autoclave. Sections were incubated overnight at room temperature with primary antibody, diluted in antibody diluent (cat\# kpxxabb500, immunologic). For primary antibodies details and dilutions, see Table 2. Omission of the primary antibodies was taken along as negative controls. Positive controls consisted of $5 \mu \mathrm{m}$ thick paraffin sections of hippocampal regions of $\mathrm{AD}$ patients. Sections were incubated for 30 min with envision (cat\# 5007, DAKO). 3,3'-Diaminobenzine (DAB) was used for color development. Nuclear counterstaining consisted of Mayers hematoxylin. Sections were dehydrated and cover slipped using quick-D (cat\# 7281, Klinipath). 

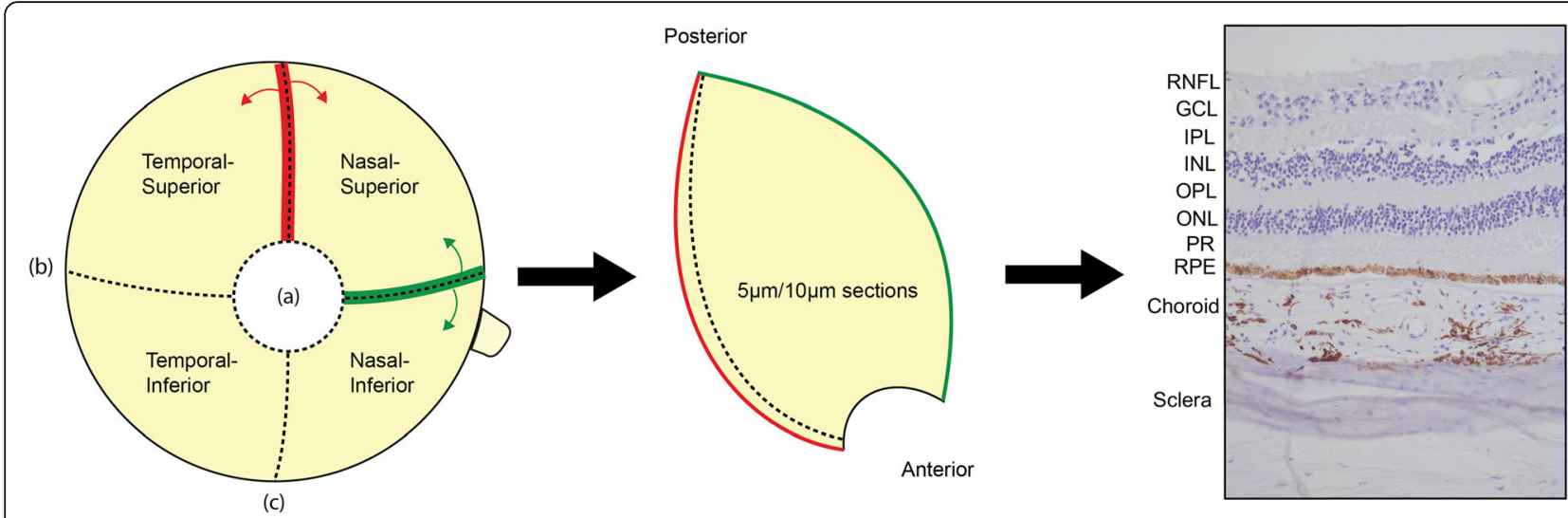

Fig. 1 Processing of post-mortem eyes. Anterior parts of eyes were removed (a). Formalin fixed eyes were dissected through the horizontal (b) and vertical meridian (c). Superior (red, arrows) and nasal (green, arrows) parts were cut in $10 \mu \mathrm{m}$ sections from anterior to posterior. As a result, sections contained all retinal layers from ora serrata to the posterior pole: retinal nerve fiber layer (RNFL), ganglion cell layer (GCL), inner plexiform layer (IPL), inner nuclear layer (INL), outer plexiform layer (OPL), outer nuclear layer (ONL), photoreceptors (PR), retinal pigment epithelium (RPE), choroid and sclera

Alternatively, when liquid permanent red (LPR) was used as chromogen, sections were incubated for $30 \mathrm{~min}$ with goat anti-mouse alkaline phosphatase (AP) (1:250, cat\# d0456, DAKO) at room temperature, after incubation with the primary antibody. After washing 3 times with TRIS buffered saline (TBS) sections were incubated with LPR (cat\#0640, DAKO), substituted with $1 \mathrm{mM}$ levamisole (to block endogenous alkaline phosphatase from blood vessels), until signal could be observed microscopically. Nuclear counterstaining was performed using Mayer's hematoxylin. Section were cover-slipped using aquatex (cat\#1.08562.0050, Merck).

\section{Curcumin}

Curcumin (cat\# 9386, Sigma Aldrich) was dissolved in $0.5 \mathrm{M} \mathrm{NaOH}$ to a stock concentration of $20 \mathrm{mg} / \mathrm{ml}$ and diluted to a final concentration of $0.1 \mathrm{mg} / \mathrm{ml}$ curcumin using PBS. Deparaffinized and rehydrated sections were incubated with $0.1 \mathrm{mg} / \mathrm{ml}$ curcumin for $10 \mathrm{~min}$ at room

Table 2 Primary antibodies used in this study

\begin{tabular}{|c|c|c|c|c|}
\hline Group & Name & Epitope & Source & Concentration \\
\hline \multirow[t]{4}{*}{$A P P / A \beta$} & $12 \mathrm{~F} 4$ & Amyloid-beta ${ }_{1-42}$ & Biolegend San Diego, CA, USA & $1: 100$ \\
\hline & $6 \mathrm{E} 10$ & Amyloid-beta ${ }_{1-16}$ & Covance, BioLegend, San Diego, CA, USA & $1: 800$ \\
\hline & $4 \mathrm{G} 8$ & Amyloid-beta $17-24$ & Biolegend, San Diego, CA & $1: 8000$ \\
\hline & APP & $\begin{array}{l}\text { APP Y188, c-terminal } \\
\text { (aa 750) }\end{array}$ & ABCAM, Cambridge, UK & $1: 6000$ \\
\hline \multirow[t]{6}{*}{ Tau } & HT7 & $\begin{array}{l}\text { Tau between residue } \\
159 \text { and } 163\end{array}$ & Invitrogen, Thermo Fisher Scientific, MA, USA & $1: 8000$ \\
\hline & AT-270 & $\begin{array}{l}\text { Tau phosphorylated at } \\
\text { Thr } 181\end{array}$ & Thermo Scientific, MA, USA & $1: 100$ \\
\hline & AT8 & $\begin{array}{l}\text { Tau phosphorylated at } \\
\text { Ser 202, Thr205 }\end{array}$ & Innogenetics, Ghent, Belgium & $1: 800$ \\
\hline & AT-100 & $\begin{array}{l}\text { Tau phosphorylated } \\
\text { Ser 212, Thr } 214\end{array}$ & Thermo Scientific, MA, USA & 1:1000 \\
\hline & Anti-pS422 & Tau Fibrils & ABCAM, Cambridge, UK & $1: 400$ \\
\hline & $M C-1$ & $\begin{array}{l}\text { Paired helical } \\
\text { filaments }\end{array}$ & $\begin{array}{l}\text { Peter Davies, Pathology and Neuroscience, } \\
\text { Donald and Barbara Zucker School of Medicine, } \\
\text { Northwell, NY, USA [21] }\end{array}$ & $1: 1000$ \\
\hline \multirow[t]{3}{*}{ Other } & Curcumin & Fibrillar $A \beta$ and CAA & Sigma Aldrich, St. Louis, MO, USA & $0,1 \mathrm{mg} / \mathrm{ml}$ in $\mathrm{NaOH}$ \\
\hline & Thioflavin & Amyloid & Sigma Aldrich, St. Louis, MO, USA & $1 \%$ in distilled water \\
\hline & $\begin{array}{l}\text { Kluver- } \\
\text { PAS }\end{array}$ & Polysaccharides & $\begin{array}{l}\text { Kluver: Luxol fast blue, BDH laboratory supplies, } \\
\text { Avantor, PA, USA, PAS: Schiff's reagens, WWR, } \\
\text { Avantor, PA, USA }\end{array}$ & $0.1 \%$ in alcohol $96 \%$ n.a. \\
\hline
\end{tabular}


temperature, washed with PBS for 3 times $10 \mathrm{~min}$ and covered using TBS/glycerol mounting medium.

\section{Thioflavin-S}

Thioflavin-S staining was performed by incubating deparaffinized and rehydrated sections with $1 \%$ thioflavin-S (cat\# T1897, Sigma Aldrich) in distilled water for $1 \mathrm{~min}$, followed by removal of excess thioflavin-S using 70\% alcohol. Stained sections were covered using TBS/glycerol mounting medium.

\section{Kluver-PAS}

Deparaffinized and rehydrated sections were incubated with $0.1 \%$ luxol fast blue (cat\# 3404438, BDH laboratory supplies) overnight at $37^{\circ} \mathrm{C}$. Sections were subsequently rinsed in ethanol $96 \%$ and distilled water. Differentiation was performed by rinsing the slides in $0.1 \%$ lithium-carbonate, followed by $70 \%$ ethanol and distilled water. Subsequently, the sections were incubated with $0.8 \%$ periodic acid for $10 \mathrm{~min}$, followed by rinsing in tap water and distilled water. Sections were incubated with Schiff's reagent (cat\# 30969.261, VWR) for $30 \mathrm{~min}$, followed by washing in tap water. Kluver-PAS stained sections were counterstained using Mayer's hematoxylin, quickly dehydrated using alcohol and xylene and covered using quick-D.

\section{PAS-IHC double staining}

Deparaffinized and rehydrated sections were incubated and $D A B$ stained as described above. DAB stained sections were washed in distilled water and incubated with $0.8 \%$ periodic acid for $10 \mathrm{~min}$. Subsequently, sections were incubated with Schiff's reagent for $30 \mathrm{~min}$, washed with tap water and counterstained using Mayer's hematoxylin. Stained sections were quickly dehydrated using ethanol and xylene and covered using quick-D.

\section{Fluorescent co-stainings with curcumin}

Sections were deparaffinized and treated with citrate buffer as described above. Subsequently, curcumin staining was performed as described above. After curcumin staining, sections were washed 3 times for $10 \mathrm{~min}$ using PBS. Primary antibody was incubated overnight at room temperature. Sections were washed 3 times with PBS and incubated with fluorescently-labelled secondary antibody (1:250, alexa 594, cat\# A11037, Invitrogen) for $3 \mathrm{~h}$. After labelling the sections were washed in PBS and covered using DAPI containing mounting medium (cat\# 0100-20, Southern Biotech).

\section{Imaging}

IHC sections were imaged by an Olympus BX41 photomicroscope using Leica Application Suite (LAS) AF lite software (Wetzlar, Germany). Fluorescently stained sections were imaged by a Leica DMi8 fluorescence microscope using LAS AF lite and ImageJ software (National Institute of Health, USA). Spectra of the Kluver-DAB double stainings were analysed using a nuance spectral imager (Perkin Elmer). Colors were assigned to binary spectra images using ImageJ.

\section{Quantification tau immunohistochemistry}

For quantitative assessment sections were photographed at four regions of interest (ROI's) from ora serrata to posterior pole of medial and superior cross-sections for each patient using an Olympus BX41 photomicroscope. Dimensions of ROI's were $316 \mu \mathrm{m} \times 115 \mu \mathrm{m}$. The DAB positive surface area (\%) within the neuroretina was quantified using the color threshold plugin in ImageJ. Between group differences were assessed using a Mann-Whitney $U$ test, using SPSS (version 22.0).

\section{Results}

Immunohistochemical detection of amyloid-beta and APP in the retina

Medial and superior retinal cross-sections were immunostained for $A \beta$-plaque pathology using anti-A $\beta$ antibodies $6 \mathrm{E} 10$ and $12 \mathrm{~F} 4$. Retinas stained with $6 \mathrm{E} 10$ showed intracellular positivity in specific retinal cell-types including ganglion cells (Fig. 2c), cells in the upper and lower portion of the inner nuclear layer (INL), most likely reflecting amacrine- and horizontal cells respectively (Fig. 2e,f), and Müller cells (Fig. 2g). In addition, staining in vessel walls (Fig. 2d) and in drusen was observed (Fig. 2h). Small extracellular deposits $(5-20 \mu \mathrm{m})$ were incidentally seen in the ganglion cell layer (GCL) and inner plexiform layer (IPL), with a predilection for the superior retina (Fig. $2 \mathrm{~b}$ and Fig. 3a). These deposits were small $(5-20 \mu \mathrm{m})$, often rounded and were morphologically different from cored plaques, diffuse plaques or fibrillary plaques observed in $\mathrm{AD}$ brain tissue. Retinas immunostained with 12F4 showed intracellular staining of ganglion cells as well (with a granular aspect), staining in vessel walls and immunostaining of small extracellular deposits in the GCL and IPL, similar as observed with 6E10 staining (Fig. 3a). Above described immunopositive features using antibodies $6 \mathrm{E} 10$ and 12F4 were more apparent in the superior part of the retina then in the medial part. Global assessment of the above described features in cross-sections of 6 control and $6 \mathrm{AD}$ cases did not separate $\mathrm{AD}$ from control cases (Fig. 3a).

In addition to $A \beta$, the antibody $6 \mathrm{E} 10$ also detects full length APP [16, 35]. Using an antibody directed against APP (C-terminal amino acid (aa) 750), similar staining as compared with $6 \mathrm{E} 10$ was seen in ganglion cells, amacrine cells, horizontal cells and Müller cells (Fig. 3a), but 


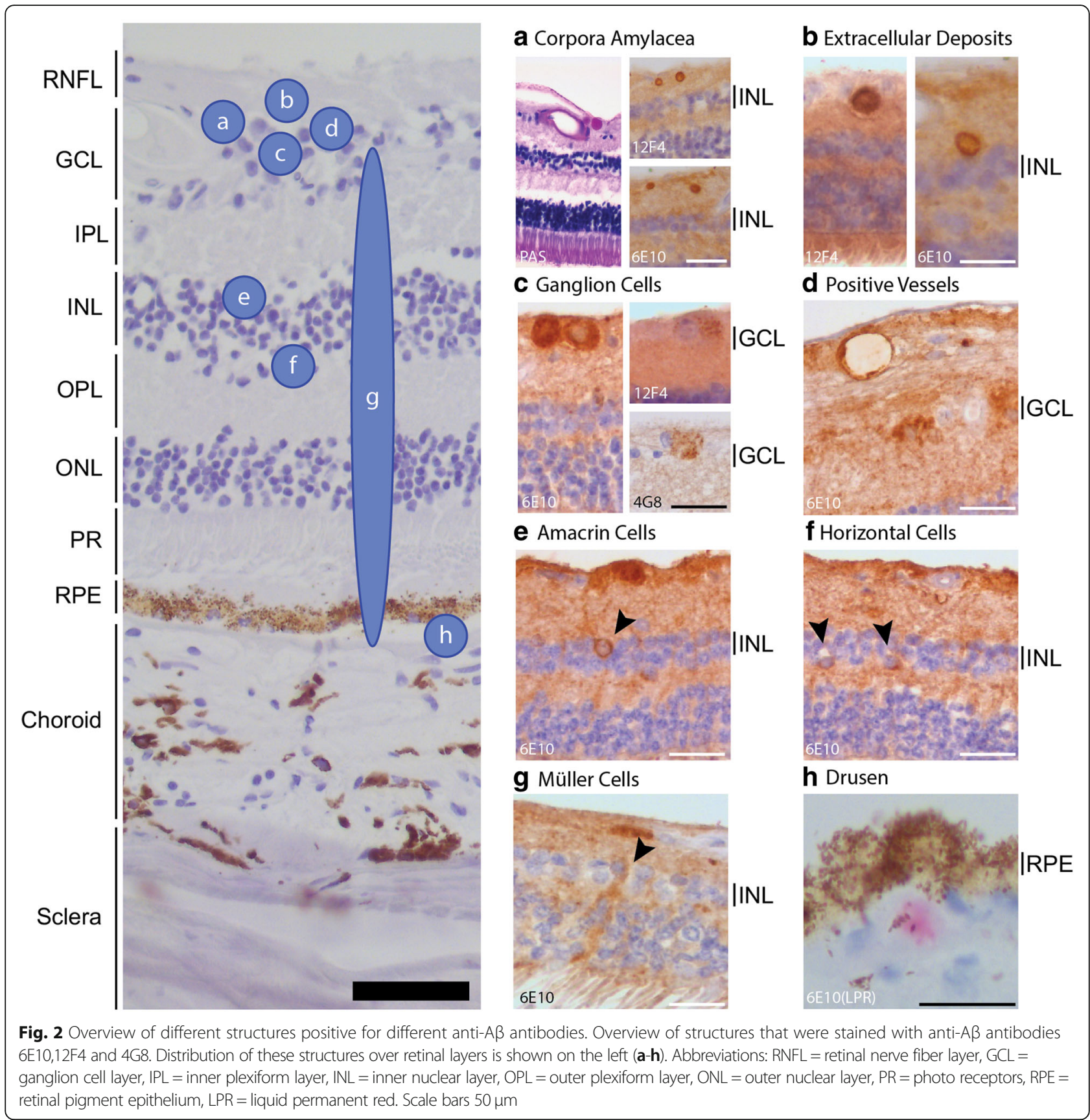

not in extracellular and vascular deposits. Subsequently, as extracellular and vascular positivity was not observed after APP immunostaining, we expanded our stainings with anti-A $\beta$ antibodies with less affinity for APP. Anti-A $\beta$ antibody $4 \mathrm{G} 8$ showed no extracellular positivity or positivity in blood vessels in $\mathrm{AD}$ or control retinas (Fig. 3a). Incidentally, granular staining of ganglion cells was observed with 4G8 (Fig. 2c). Lastly, additional staining with Thioflavin-S, did not show positive staining, while plaques and tangles were observed in $\mathrm{AD}$ hippocampal tissue (Fig. 3a).
In summary, intracellular staining was observed with 12F4 and 6E10 and APP antibodies. In addition, 6E10 and 12F4 antibodies showed small extracellular deposits that were negative for $4 \mathrm{G} 8$ and APP antibodies.

\section{Corpora amylacea are detected in $A D$ and control retina}

We investigated whether the small 6E10/12F4 positive, 4G8 negative deposits, could be related to corpora amylacea, deposits associated with aging as well as neurodegeneration [29]. We therefore assessed these deposits using a combination of 6E10 antibody and Kluver-PAS 


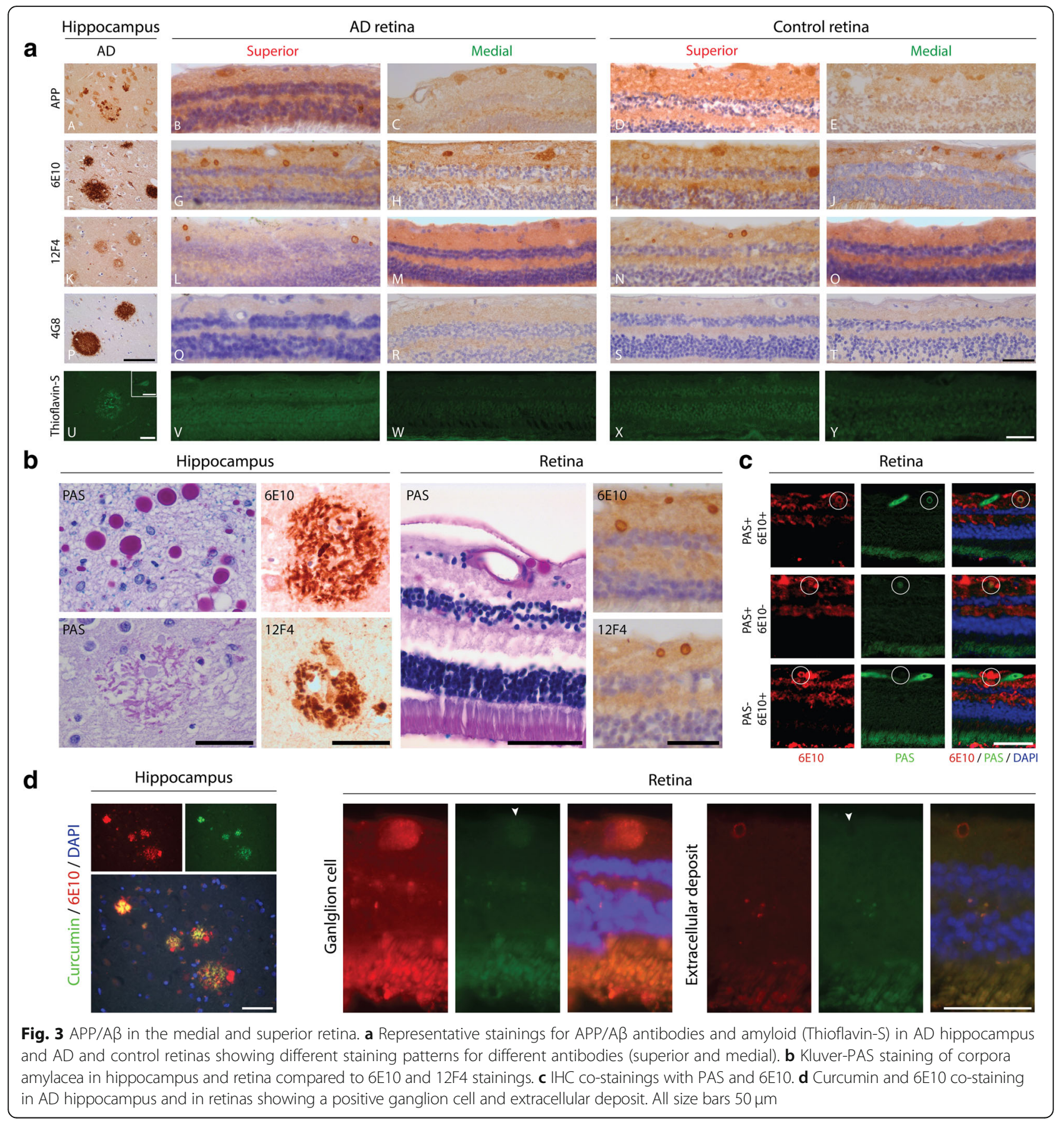

staining, a histochemical staining routinely used for the detection of corpora amylacea. With the Kluver-PAS we observed staining of corpora amylacea in $\mathrm{AD}$ brain tissue, while plaques were not or only faintly labeled (Fig. 3b). Retinas of both AD and controls showed extracellular, rounded depositions with the Kluver-PAS staining, that were comparable in size and localization to the structures observed with the $6 \mathrm{E} 10$ and $12 \mathrm{~F} 4$ antibodies in adjacent retinal cross-sections (Fig. 3b).
Next we performed double-labelling combining the Kluver-PAS staining with immunodetection with the $6 \mathrm{E} 10$ antibody. A subset of $6 \mathrm{E} 10$ positive deposits co-localized with Kluver-PAS positive deposits, while others were Kluver-PAS negative (Fig. 3c). In addition, we also observed PAS positive, 6E10 negative deposits (Fig. 3c).

To assess fibrillarity of extracellular deposits and enable translation of our findings to in-vivo imaging with 
curcumin, we performed Thioflavin-S and curcumin stainings of adjacent sections [8]. No extracellular deposits were detected with curcumin or Thioflavin-S (Fig. $3 \mathrm{a}$ and $\mathrm{d}$ ). This finding was confirmed by fluorescent co-stainings with 6E10 and curcumin (Fig. 3d). Fluorescent co-stainings with $6 \mathrm{E} 10$ and curcumin did show co-localization of (faint) curcumin staining with 6E10 intracellularly (Fig. 3d).

In summary, we observed corpora amylacea in $\mathrm{AD}$ and control retina that explained a proportion of small 6E10 immunopositive extracellular deposits. These extracellular deposits were negative for Thioflavin or curcumin.

\section{Tau and phosphorylated tau in AD and control retina}

Next to amyloid pathology, AD is characterized by tau pathology, which encompasses accumulation of phosphorylated tau (pTau) in neuropil threads, neurofibrillary tangles (NFTs) and neuritic plaques, primarily in cortical areas of the brain [31]. We therefore assessed presence of tau and its phosphorylated isoforms in $\mathrm{AD}$ and control retinas. As reported previously [25], total tau assessed with antibody HT7 shows tau distribution in three distinct layers in the inner plexiform layer (IPL) and diffusely in the outer plexiform layer (OPL) (Fig. 4). Diffuse axonal positivity in IPL and OPL was observed with anti-pTau antibodies AT8, AT100 and AT270
(Fig. 4). Overall, more immunoreactivity for AT8, AT100 and AT270 was present in AD cases compared to controls (Fig. 5). However, no structures related to NFTs, neuropil threads or neuritic plaques, as observed in the cerebral cortex, were observed with these antibodies in $\mathrm{AD}$ or control retinas. pTau (AT8) staining was more apparent in superior than in medial parts of the retina and showed a positive gradient towards the periphery (Fig. 5). Two controls with an amyloid Braak stage B and C showed similar pTau (AT8) patterns as observed in the retina of $\mathrm{AD}$ cases. Table 3 shows quantification of AT8 immunoreactivity in medial and superior retinas of $\mathrm{AD}$ versus control cases. Mean surface of AT8 immunopositivity in medial and superior parts of the retina did not statistically differ between $\mathrm{AD}$ cases and controls (Mann-Whitney $\mathrm{U}$ test $p=0.310$ and $p=0.537$ respectively), as two controls with moderate to severe amyloid pathology were considerable outliers. Stratified for amyloid stage, cases with a moderate to severe amyloid stage $(\mathrm{B}, \mathrm{C})$ showed higher AT8 immunopositivity compared to cases with low amyloid stages $(\mathrm{O}, \mathrm{A})$ in medial (Mean $5.28 \%$ $( \pm 9.83)$ vs. $0.01 \%( \pm 0.01)$, Mann-Whitney $\mathrm{U}$ test, $p=0.004)$ and superior parts of the retina (Mean $12.14 \%( \pm 15.60)$ vs. $0.06 \%( \pm 0.08)$, Mann-Whitney $\mathrm{U}$ test, $p=0.012)$. No immunostaining in control and $\mathrm{AD}$ retina was observed with anti-tau pS422 or antibody MC-1, which specifically detects paired helical filaments (Fig. 4).

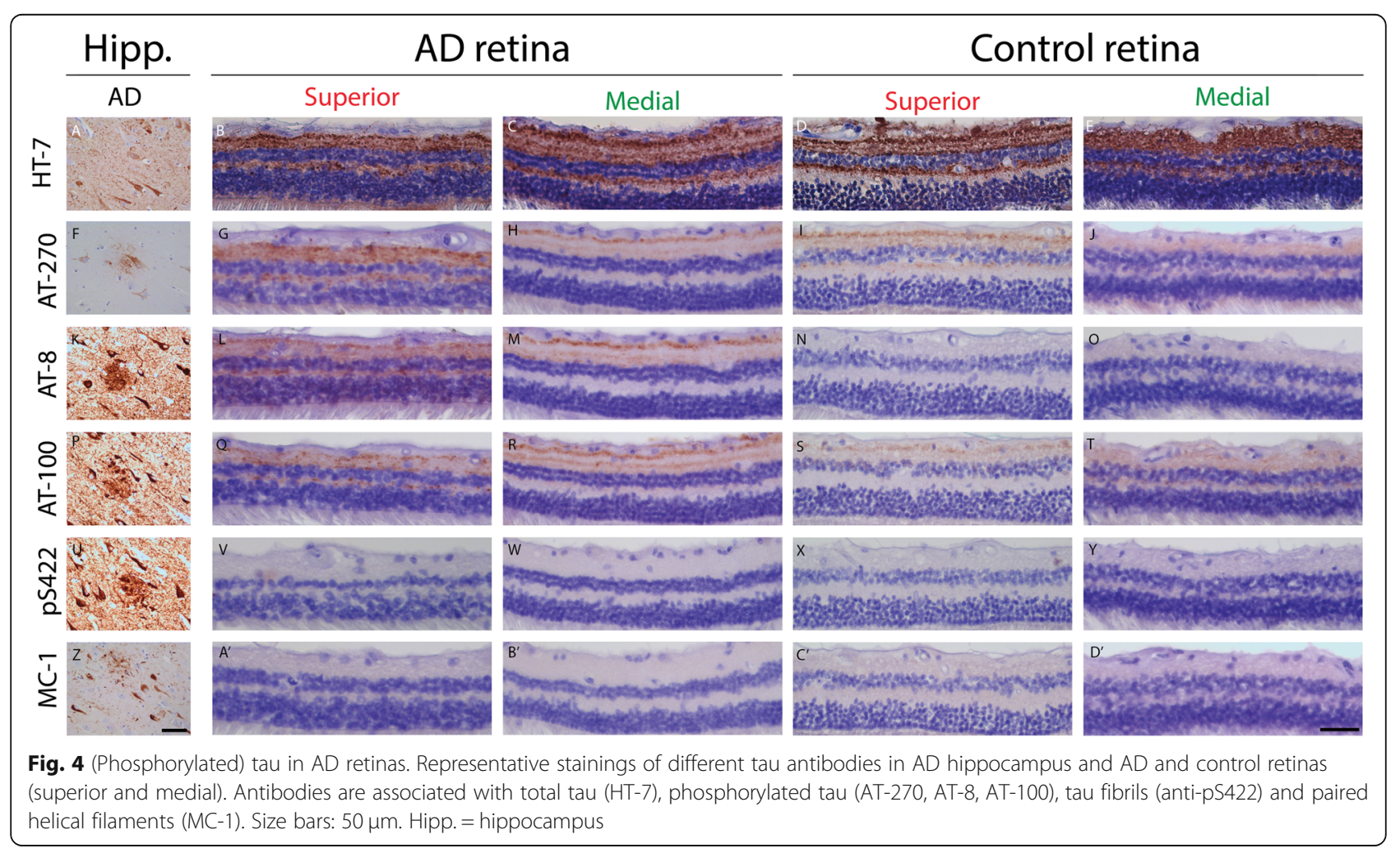




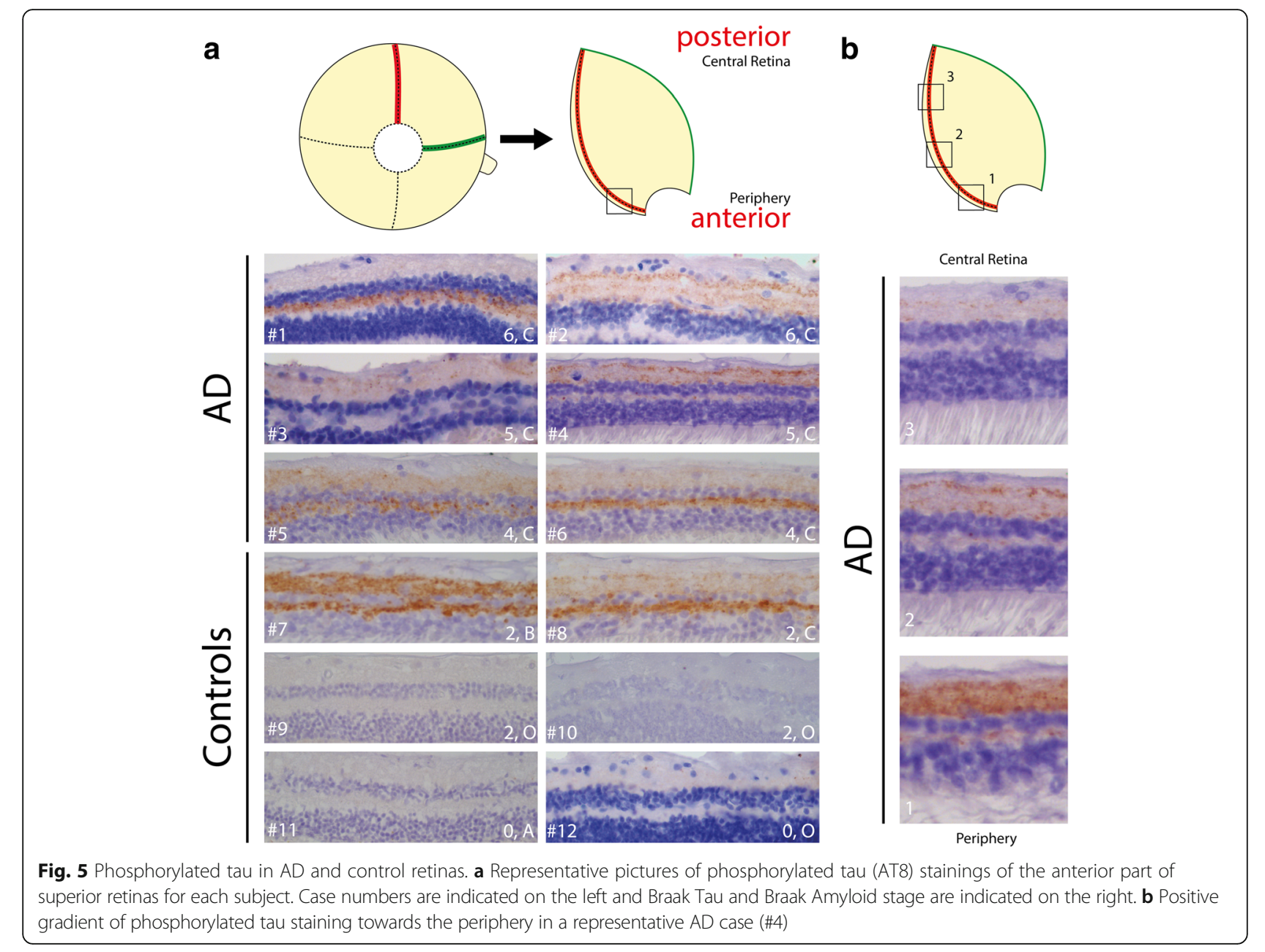

In summary, diffuse phosphorylated tau for three phosphorylation sites was observed in $\mathrm{AD}$, with a predilection for the peripheral retina, while NFTs, neuritic plaques, fibrillar tau or paired helical filaments were not detected.

\section{Discussion}

In this post-mortem study of well-characterized $\mathrm{AD}$ and control cases, we qualitatively assessed antibody panels for APP, A $\beta$ and tau on AD and control retinal crosssections. We found that diffuse phosphorylated tau in the retina separated $\mathrm{AD}$ cases from controls while immunoreactivity for APP and A $\beta$ in the retina did not differ between groups.

In order to resolve discrepancies between studies reporting retinal $A \beta$ we, for the first time, assessed the presence of APP and A $\beta$ in the retina using a wide panel of antibodies. Our results implicate that APP/A $\beta$ pathology in the retina does not clearly separate AD cases from controls. Using an APP antibody we showed that a large proportion of immunostaining with $6 \mathrm{E} 10$ and 12F4 showed overlap with, and could be explained by, intracellular staining of APP in retinal cell types that are known to express APP [28]. In addition, it could also represent intraneuronal $\beta$-secretase cleaved APP $\beta$-C-terminal fragments (CTFs) including C83, C99 and AICD [11], as our APP antibody binds to the C-terminal of APP (aa750). We hypothesize that intracellular APP/A $\beta$ might reflect metabolic activity in different retinal cell populations expressing APP, as reported for ganglion cells and INL cells [28], and (efficient) processing of $\mathrm{APP} / \mathrm{A} \beta$ to the outer retina by Müller cells, cells that are responsible for retinal homeostasis [32]. High levels of APP in the absence of clear extracellular fibrillar $A \beta$ deposits suggests that the amyloidogenic and non-amyloidogenic pathway are differently controlled in the retina, compared to the brain. In addition, the build-up of fibrillar $A \beta$ might be confined to the intracellular compartment in the retina. Assessment of mechanisms controlling the amyloidogenic and non-amyloidogenic pathways in the retina is therefore needed to assess the precise role of $A \beta$ processing in the retina in $\mathrm{AD}$ and aging. Understanding these mechanisms the role of retinal amyloid as non-invasive biomarker, but could also yield information on selective vulnerability or resilience of specific neuronal populations [9]. 
Table 3 Quantification of phosporylated Tau (AT8) Positivity

\begin{tabular}{|c|c|c|c|c|c|c|c|}
\hline \multirow[t]{3}{*}{$\#$} & \multirow{3}{*}{$\begin{array}{l}\text { Pathological } \\
\text { diagnosis }\end{array}$} & \multirow{3}{*}{$\begin{array}{l}\text { Braak } \\
\text { Tau }\end{array}$} & \multirow{3}{*}{$\begin{array}{l}\text { Braak } \\
\text { Amyloid }\end{array}$} & \multicolumn{4}{|c|}{ AT8 positivity (\% surface area) } \\
\hline & & & & \multicolumn{2}{|c|}{ Superior } & \multicolumn{2}{|l|}{ Medial } \\
\hline & & & & Mean & $\mathrm{sd}$ & Mean & $\mathrm{sd}$ \\
\hline 1 & $A D$ & $\mathrm{Vl}$ & C & 3,41 & 2,82 & 1,88 & 0,44 \\
\hline 2 & $A D$ & $\mathrm{Vl}$ & C & 0,79 & 0,70 & 1,07 & 0,55 \\
\hline 3 & $A D$ & V & C & 1,35 & 0,38 & 0,04 & 0,05 \\
\hline 4 & $A D$ & V & C & 15,36 & 5,13 & 4,12 & 2,52 \\
\hline 5 & $A D$ & IV & C & 6,87 & 1,02 & 0,37 & 0,01 \\
\hline \multirow[t]{2}{*}{6} & $A D$ & IV & C & 8,72 & 1,65 & 1,97 & 1,25 \\
\hline & & & Mean & 6.08 & 5.50 & 1.57 & 1.47 \\
\hline 7 & $\mathrm{HC}$ & $\|$ & B & 48,65 & 8,73 & 29,35 & 2,62 \\
\hline 8 & $\mathrm{HC}$ & $\|$ & C & 11,92 & 1,84 & 3,43 & 1,87 \\
\hline 9 & $\mathrm{HC}$ & $\|$ & O & 0,15 & 0,18 & 0,00 & 0,01 \\
\hline 10 & $\mathrm{HC}$ & $\|$ & O & - & - & 0,01 & 0,01 \\
\hline 11 & $\mathrm{HC}$ & 0 & A & 0,02 & 0,02 & 0,03 & 0,02 \\
\hline \multirow[t]{2}{*}{12} & $\mathrm{HC}$ & 0 & O & 0,01 & 0,01 & 0,00 & 0,00 \\
\hline & & & Mean & 12.15 & 21.04 & 5.50 & 11.78 \\
\hline
\end{tabular}

Means and standard deviations (sd) of AT8 positivity (\% surface area) of four superior and medial pictures per patient. $A D$ Alzheimer's disease, $H C$ Healthy control

We replicated earlier findings of small extracellular deposits positive for $6 \mathrm{E} 10$ and $12 \mathrm{~F} 4$ antibodies in the RNFL and GCL with a predilection to the superior retina [22]. Morphologically these deposits were different from $A \beta$-plaques observed in the cortex and hippocampus, smaller and also observed in control cases. Using a wide panel of anti-A $\beta$ and APP antibodies we further characterized these deposits, considering cross-reactivity towards A $\beta$ and APP by these antibodies [16]. Interestingly, observed deposits were negative for an anti-A $\beta$ antibody with relatively less affinity for APP (4G8). In addition, an anti-APP antibody directed at the C-terminal (aa 750) did not show immunoreactivity towards these extracellular deposits. As 6E10 is directed to $A \beta_{3-}$ 10 and has affinity for APP, a possible explanation is that 6E10 recognizes an APP isoform that is not detected by the used APP antibody, for instance the soluble APP $\alpha$ isoform that lacks the C-terminal part of APP. Extracellular immunopositivity with the $12 \mathrm{~F} 4$ antibody could be explained by detection of IgMs by $12 \mathrm{~F} 4$ as described in previous literature [2]. Using IHC co-stainings with Kluver-PAS, we showed that a proportion of these deposits can be characterized as corpora amylacea, deposits previously associated with aging and neurodegeneration in the brain [29]. Finally, the absence of co-staining of these deposits with curcumin and Thioflavin suggests absence of fibrillar structures, like fibrillar $A \beta$ [8], and questions whether these deposits can be visualized in-vivo using curcumin [22].
Secondly, we assessed the presence of tau, its phosphorylated isoforms, fibrillar tau (pS422) and paired helical filaments (MC-1). Using antibodies AT8, AT100 and AT270 directed at different phospho-epitopes of tau revealed a diffuse signal in the inner plexiform layer (IPL) and outer plexiform layer (OPL) of $\mathrm{AD}$ cases, while NFT's, neuropil threads and neuritic plaques were not observed. In addition, we observed no fibrillar tau or paired helical filaments. A previous report by Schön and coworkers also showed a diffuse signal of pTau with AT8, while phosphorylation sites detected by AT100, AT180 and AT270 yielded negative results [30]. In addition, similar to our study no fibrillary tau was observed, while in contrast to our findings, intracellular inclusions (NFTs) were observed in 5 out of $6 \mathrm{AD}$ cases using the AT8 antibody. Discrepancies with this study may be explained by differences in antibody dilutions, retinal regions studied and differences in expression of retinal pathology between $\mathrm{AD}$ patients.

The diffuse signal of pTau observed in the current study was most apparent in the peripheral retina, as reported previously in tau transgenic mice [15]. This, might be of interest for in-vivo studies that currently mainly focus on imaging of the central retina. Recent developments in imaging technology make it possible to perform in-vivo peripheral imaging of the retina. This may be a promising step forward in visualizing $\mathrm{AD}$ pathology in the peripheral retina [27].

Interestingly, two cognitively normal controls with moderate amyloid pathology showed intense tau staining patterns similar to $\mathrm{AD}$ cases. This might suggest early involvement of tau-pathology in the retina in AD. Mechanistically, this could be the result of local pathology or transneuronal propagation of phosphorylated tau originating in the visual cortices [10]. Supporting the latter, both control cases had moderate to severe amyloid pathology in their visual cortices that could have already contributed to local toxicity to dendrites and subsequent tau phosphorylation [18]. On the other hand, caution is warranted for the (over)interpretation of these findings, as tau phosphorylation might also be the result of confounding (retinal) pathology, including glaucoma [12] or an aging effect. Supporting the latter, we observed that a young onset AD case (case \#4, 56 years) showed little pTau immunopositivity, while two older controls (case \#7 89 years, case \#8 80 years) showed apparent diffuse pTau immunopositivity. Future studies in larger cohorts, including patients with a range of Braak and amyloid stages, and considering age as confounder, are needed to assess pTau as a possible AD biomarker in the retina. Studies on different tauopathies are needed to assess the specificity of these findings and the possible use of retinal tau as biomarker in other tauopathies and glaucoma. Finally, development of fluorescent labels for 
phosphorylated tau for human use is needed in order to translate these findings to an in-vivo set-up using targeted fluorescence. A recent proof of concept study showed the possibility of molecular imaging using targeted fluorescence, by in-vivo labeling of single-cell apoptosis in glaucoma [5].

Important strengths of this study are the use of well-characterized cases and controls, and the use of antibody panels on sequential cross-sections, permitting a qualitative assessment of retinal AD pathology and its layer distribution. Despite the fact that we aimed to address sampling bias by staining a large number of $10 \mu \mathrm{m}$ thick sections per patient, we should note that we might have missed infrequent pathology, including the temporal and inferior retinal regions. Using flat-mounts in future studies to complement a cross-sectional approach could help overcome sampling bias. Using such an approach, inherently lacking qualitative layer information, caution should be taken that $6 \mathrm{E} 10$ and $12 \mathrm{~F} 4$ positivity includes immunoreactivity towards intracellular APP, corpora amylacea and drusen. Future studies are needed to assess if (peripheral) drusen could represent an increased $\mathrm{A} \beta$ reservoir in aging and $\mathrm{AD}$. In addition, for this study a relatively small sample size was assessed using a qualitative approach to assess neuropathological hallmarks of $\mathrm{AD}$ in the retina. Disease effects are typically observed in comparable cohorts. However, to further understand observed effects and study whether more subtle changes are present in the retina larger cohorts should be assessed using a quantitative approach.

\section{Conclusion}

Our findings confirm the presence of phosphorylated tau in the retina, nuance earlier findings of retinal $A \beta$ and explain negative findings from previous studies, using antibodies with less APP affinity. Independent studies using comparable protocols for tissue processing and staining are needed to resolve discrepancies between different labs [14, 22, 30, 34]. These studies could help determine which of the observed retinal changes are age-related and which are disease related and could serve as a target for in-vivo imaging. In conclusion, neuropathological hallmarks of AD appear differently in the retina compared to the cerebral cortex. Diffuse phosphorylated tau in the peripheral retina might be a promising target for in-vivo imaging.

\footnotetext{
Abbreviations

${ }^{\circ} \mathrm{C}$ : Celsius; AD: Alzheimer's disease; APP: Amyloid precursor protein; AB: Amyloid-beta; CAA: Cerebral amyloid angiopathy; CERAD: Consortium to establish registry for Alzheimer's disease; DAB: 3,3'-diaminobenzidine; DAPI: 4'6-diamidino-2-phenylindole; GCL: Ganglion cell layer; IHC: Immunohistochemistry; INL: Inner nuclear layer; IPL: Inner plexiform layer; LPR: Liquid permanent red; NBB: The Netherlands brain bank; NFTs: Neurofibrillary tangles; NIA-AA: National Institute of Aging and Alzheimer's Association; OPL: Outer plexiform layer; PAS: Periodic acid
}

Schiff; PBS: Phosphate-buffered saline; PFA: Paraformaldehyde; PR: Photo receptors; pTau: Phosphorylated tau; RNFL: Retinal nerve fiber layer; RPE: Retinal pigment epithelium; TBS: Tris-buffered saline

\section{Acknowledgements}

Anti-paired helical filaments antibody (MC-1) was kindly provided by Peter Davies, Pathology and Neuroscience, Donald and Barbara Zucker School of Medicine, Northwell, NY, USA. We kindly thank the Netherlands Brain Bank, J.B. ten Brink and N.P. Smoors, for their contributions to this work.

\section{Funding}

This research was funded by Alzheimer Nederland (WE.09-2016-03) and by the Dutch Technology Foundation STW (grant number 13935), which is part of the Netherlands Organisation for Scientific Research (NWO), and which is partly funded by the Ministry of Economic Affairs.

\section{Availability of data and materials}

Not applicable.

\section{Authors' contributions}

$\mathrm{JdH}$, designed the study and experiments, performed analysis, prepared figures and drafted the manuscript. THJM designed experiments, performed the experiments, performed analysis and drafted the manuscript. JJH designed the study, performed analysis and supervised the study. FHB designed and supervised the study. All authors read and approved the final manuscript.

\section{Ethics approval and consent to participate}

Prior to death, donors signed informed consent with the Netherlands Brain Bank (NBB) for brain autopsy and use of brain tissue and medical records for research purposes.

\section{Consent for publication}

Not applicable.

\section{Competing interests}

The authors declare that they have no competing interests.

\section{Publisher's Note}

Springer Nature remains neutral with regard to jurisdictional claims in published maps and institutional affiliations.

\section{Author details}

${ }^{1}$ Department of Neurology, Alzheimer Center Amsterdam, Amsterdam Neuroscience, Vrije Universiteit Amsterdam, Amsterdam UMC, Mailbox 7057, Amsterdam 1007 MB, The Netherlands. ²Department of Pathology, Amsterdam Neuroscience, Amsterdam UMC, Vrije Universiteit Amsterdam, Amsterdam, The Netherlands. ${ }^{3}$ Ophthalmology Department, VU University Medical Center, Amsterdam, The Netherlands. ${ }^{4}$ Department of Physics, Bio Laser Lab Amsterdam, VU University, Amsterdam, The Netherlands. ${ }^{5}$ Departments of Clinical genetics and Ophthalmology, Amsterdam UMC, Academic Medical Center, University of Amsterdam, Amsterdam, The Netherlands. ${ }^{6}$ The Netherlands Institute for Neuroscience (NIN-KNAW), Amsterdam, The Netherlands.

Received: 6 December 2018 Accepted: 11 December 2018

Published online: 28 December 2018

References

1. Association As (2018) Alzheimer's disease facts and figures. https:// wwwalzorg/documents_custom/2018-facts-and-figurespdf:

2. Auge E, Cabezon I, Pelegri C, Vilaplana J (2017) New perspectives on corpora amylacea in the human brain. Sci Rep 7:41807. https://doi.org/10. 1038/srep41807

3. Blanks JC, Torigoe Y, Hinton DR, Blanks RH (1996) Retinal pathology in Alzheimer's disease. I. Ganglion cell loss in foveal/parafoveal retina. Neurobiol Aging 17:377-384

4. Braak H, Braak E (1991) Neuropathological stageing of Alzheimer-related changes. Acta Neuropathol 82:239-259.

5. Cordeiro MF, Normando EM, Cardoso MJ, Miodragovic S, Jeylani S, Davis BM, Guo L, Ourselin S, A'Hern R, Bloom PA (2017) Real-time 
imaging of single neuronal cell apoptosis in patients with glaucoma. Brain 140:1757-1767. https://doi.org/10.1093/brain/awx088

6. Cummings JL (2004) Alzheimer's disease. N Engl J Med 351:56-67. https:// doi.org/10.1056/NEJMra040223

7. Davis BM, Crawley L, Pahlitzsch M, Javaid F, Cordeiro MF (2016) Glaucoma: the retina and beyond. Acta Neuropathol 132:807-826. https://doi.org/10. 1007/s00401-016-1609-2

8. den Haan J, Morrema THJ, Rozemuller AJ, Bouwman FH, Hoozemans JJM (2018) Different curcumin forms selectively bind fibrillar amyloid beta in post mortem Alzheimer's disease brains: implications for in-vivo diagnostics. Acta Neuropathol Commun 6:75. https://doi.org/10.1186/ s40478-018-0577-2

9. Fu H, Hardy J, Duff KE (2018) Selective vulnerability in neurodegenerative diseases. Nature Neuroscience. https://doi.org/10.1038/s41593-018-0221-2

10. Gibbons GS, Lee VMY, Trojanowski JQ (2018) Mechanisms of cell-to-cell transmission of pathological tau: a review. JAMA Neurol. https://doi.org/10 1001/jamaneurol.2018.2505

11. Gouras GK, Tampellini D, Takahashi RH, Capetillo-Zarate E (2010) Intraneuronal beta-amyloid accumulation and synapse pathology in Alzheimer's disease. Acta Neuropathol 119:523-541.

12. Gupta N, Fong J, Ang LC, Yucel YH (2008) Retinal tau pathology in human glaucomas. Can J Ophthalmol 43:53-60. https://doi.org/10.3129/107-185

13. Heneka MT, Carson MJ, El Khoury J, Landreth GE, Brosseron F, Feinstein DL, Jacobs AH, Wyss-Coray T, Vitorica J, Ransohoff RM et al (2015). Neuroinflammation in Alzheimer's disease. Lancet Neurol 14:388-405. https://doi.org/10.1016/s1474-4422(15)70016-5

14. Ho CY, Troncoso JC, Knox D, Stark W, Eberhart CG (2014) Beta-amyloid, phospho-tau and alpha-synuclein deposits similar to those in the brain are not identified in the eyes of Alzheimer's and Parkinson's disease patients. Brain Pathol 24:25-32. https://doi.org/10.1111/bpa.12070

15. Ho WL, Leung Y, Cheng SS, Lok CK, Ho YS, Baum L, Yang X, Chiu K, Chang RC (2015) Investigating degeneration of the retina in young and aged tau P301L mice. Life Sci 124:16-23. https://doi.org/10.1016/j.lfs.2014.12.019

16. Hunter S, Brayne C (2017) Do anti-amyloid beta protein antibody cross reactivities confound Alzheimer disease research? J Negat Results Biomed 16:1.

17. Hyman BT, Phelps CH, Beach TG, Bigio EH, Cairns NJ, Carrillo MC, Dickson DW, Duyckaerts C, Frosch MP, Masliah E et al (2012) National Institute on Aging-Alzheimer's Association guidelines for the neuropathologic assessment of Alzheimer's disease. Alzheimers Dement 8:1-13. https://doi. org/10.1016/j.jalz.2011.10.007

18. Ittner LM, Ke YD, Delerue F, Bi M, Gladbach A, van Eersel J, Wolfing $H$, Chieng BC, Christie MJ, Napier IAet al (2010) Dendritic function of tau mediates amyloid-beta toxicity in Alzheimer's disease mouse models. Cell 142: 387-397 Doi https://doi.org/10.1016/j.cell.2010.06.036

19. Jack CR, Holtzman DM (2013) Biomarker modeling of alzheimer's disease. Neuron 80:1347-1358. https://doi.org/10.1016/..neuron.2013.12.003

20. Jiang J, Wang H, Li W, Cao X, Li C (2016) Amyloid plaques in retina for diagnosis in Alzheimer's patients: a meta-analysis. Front Aging Neurosci 8:267.

21. Jicha GA, Bowser R, Kazam IG, Davies P (1997) Alz-50 and MC-1, a new monoclonal antibody raised to paired helical filaments, recognize. conformational epitopes on recombinant tau. J Neurosci Res 48:128-132

22. Koronyo Y, Biggs D, Barron E, Boyer DS, Pearlman JA, Au WJ, Kile SJ, Blanco A, Fuchs DT, Ashfaq Aet al (2017) Retinal amyloid pathology and proof-ofconcept imaging trial in Alzheimer's disease. JCl Insight 2: Doi https://doi. org/10.1172/jci.insight.93621

23. Koronyo-Hamaoui M, Koronyo Y, Ljubimov AV, Ca M, Ko MK, Black KL, Schwartz M, Farkas DL (2011) Identification of amyloid plaques in retinas from Alzheimer's patients and noninvasive in vivo optical imaging of retinal plaques in a mouse model. Neurolmage 54:S204-S217. https://doi.org/10. 1016/j.neuroimage.2010.06.020

24. La Morgia C, Ross-Cisneros FN, Koronyo Y, Hannibal J, Gallassi R, Cantalupo G, Sambati L, Pan BX, Tozer KR, Barboni P et al (2015) Melanopsin retinal ganglion cell loss in Alzheimer's disease. Ann Neurol. https://doi.org/10. 1002/ana.24548

25. Loffler KU, Edward DP, Tso MO (1995) Immunoreactivity against tau, amyloid precursor protein, and beta-amyloid in the human retina. Invest Ophthalmol Vis Sci 36:24-31.

26. Pelak VS, Hills W (2018) Vision in Alzheimer's disease: a focus on the anterior afferent pathway. Neurodeger Dis Manag 8:49-67. https://doi.org/10.2217/ nmt-2017-0030
27. Quinn N, Csincsik L, Flynn E, Curcio CA, Kiss S, Sadda SR, Hogg R, Peto T, Lengyel I (2018) The clinical relevance of visualising the peripheral retina. Prog Retin Eye Res. https://doi.org/10.1016/j.preteyeres.2018.10.001

28. Ratnayaka JA, Serpell LC, Lotery AJ (2015) Dementia of the eye: the role of amyloid beta in retinal degeneration. Eye (Lond) 29:1013-1026. https://doi. org/10.1038/eye.2015.100

29. Rohn TT (2015) Corpora Amylacea in neurodegenerative diseases: cause or effect? Int J Neurol Neurother 2(3):031. https://www.ncbi.nlm.nih.gov/ pubmed/26550607.

30. Schön C, Na H, Ochs SM, Burgold S, Filser S, Steinbach S, Seeliger MW, Arzberger T, Goedert M, Kretzschmar HA et al (2012) Long-Term In Vivo Imaging of Fibrillar Tau in the Retina of P301S Transgenic Mice. PLoS ONE 7 1-9. https://doi.org/10.1371/journal.pone.0053547

31. Spillantini MG, Goedert M (2013) Tau pathology and neurodegeneration. Lancet Neurol 12:609-622. https://doi.org/10.1016/s1474-4422(13)70090-5

32. Subirada PV, Paz MC, Ridano ME, Lorenc VE, Vaglienti MV, Barcelona PF, Luna JD, Sanchez MC (2018) A journey into the retina: Muller glia commanding survival and death. Eur J Neurosci 47:1429-1443. https://doi. org/10.1111/ejn.13965

33. Thal DR, Ghebremedhin E, Rub U, Yamaguchi H, Del Tredici K, Braak H (2002) Two types of sporadic cerebral amyloid angiopathy. J Neuropathol Exp Neurol 61:282-293.

34. Williams EA, McGuone D, Frosch MP, Hyman BT, Laver N, StemmerRachamimov A (2017) Absence of Alzheimer disease Neuropathologic changes in eyes of subjects with Alzheimer disease. J Neuropathol Exp Neurol 76:376-383. https://doi.org/10.1093/jnen/n|x020

35. Youmans KL, Tai LM, Kanekiyo T, Stine WB, Jr., Michon SC, Nwabuisi-Heath E, Manelli AM, Fu Y, Riordan S, Eimer WA et al (2012) Intraneuronal Abeta detection in 5XFAD mice by a new Abeta-specific antibody. Mol Neurodegen 7: 8 Doi https://doi.org/10.1186/1750-1326-7-8

Ready to submit your research? Choose BMC and benefit from:

- fast, convenient online submission

- thorough peer review by experienced researchers in your field

- rapid publication on acceptance

- support for research data, including large and complex data types

- gold Open Access which fosters wider collaboration and increased citations

- maximum visibility for your research: over $100 \mathrm{M}$ website views per year

At $\mathrm{BMC}$, research is always in progress.

Learn more biomedcentral.com/submissions 